\title{
Synthesis of Seven-Membered Ring Glycals via Endo-Selective Alkynol Cycloisomerization
}

Eva Alcázar, ${ }^{\dagger}$ Joseph M. Pletcher, and Frank E. McDonald*

Department of Chemistry, Emory University, Atlanta, GA 30322

\section{SUPPORTING INFORMATION}

Contents:

General information $\quad$ S-2

Alkynyl alcohol (4) S-3

Alkynyl diol (5) S-4

Dihydropyran (6) S-5

Exocyclic enol ether (7) S-5

Oxepine glycal (8) S-6

Oxepine glycal, acetate ester (9) $\quad$ S-7

Alkynyl diol (10) S-7

Oxepine glycal (11) S-8

nOe spectrum of $\mathbf{1 1}$ S-9

Alkynyl diol (12) S-10

Oxepine glycal, alcohol (13-OH) S-10

Oxepine glycal (13) (acetate ester) S-11

Alkynyl diol (14) S-11

Oxepine glycal, alcohol (15-OH) S-12

Oxepine glycal (15) (acetate ester) S-12

L-arabino-benzoate precursor to $\mathbf{1 6}$ S-13

Alkynyl diol (16) S-14

Oxepine glycal, alcohol (17-OH) S-14

Oxepine glycal (17) (acetate ester) S-15

Alkynyl diol (18) S-15

Oxepine glycal (19) (acetate ester) S-16

COSY spectrum of $\mathbf{1 9} \quad \mathrm{S}-17$

Alkynyl diol (22) S-18

Elimination product (24) S-18

COSY spectrum of $\mathbf{2 4}$ S-19 
General: ${ }^{1} \mathrm{H}$ NMR spectra were recorded at either $300 \mathrm{MHz}$ on a Varian Mercury-300, at 400 $\mathrm{MHz}$ on an Inova-400 spectrometer, or at $600 \mathrm{MHz}$ on an INOVA-600 spectrometer. ${ }^{13} \mathrm{C}$ NMR spectra were recorded at $75 \mathrm{MHz}, 100 \mathrm{MHz}$, or $150 \mathrm{MHz}$ on the same instruments. NMR spectra were recorded in deuterated chloroform $\left(\mathrm{CDCl}_{3}\right)$ solutions, with residual chloroform $(\delta$ $7.26 \mathrm{ppm}$ for ${ }^{1} \mathrm{H}$ NMR and $\delta 77.00 \mathrm{ppm}$ for ${ }^{13} \mathrm{C}$ NMR) taken as the internal standard, and were reported in parts per million (ppm). Abbreviations for signal coupling are as follows: s, singlet; d, doublet; t, triplet; q, quartet; m, multiplet. IR spectra were collected on a Mattson Genesis II FT-IR spectrometer as neat films. Elemental analyses were performed by Atlantic Microlab Inc, P. O. Box 2288, Norcross, Georgia. Analytical Thin Layer Chromatography (TLC) was performed on precoated glass backed plates purchased from Whatman (silica gel $60 \mathrm{~F}_{254}$; $0.25 \mathrm{~mm}$ thickness). Flash column chromatography was conducted with silica gel 60 (230-400 mesh ASTM) from EM Science. Optical rotations were recorded with Perkin-Elmer model 241 or 341 polarimeters.

All reactions were carried out with anhydrous solvents in oven-dried or flame-dried and argon-charged glassware. All anhydrous solvents except as mentioned were freshly distilled. All solvents used in workup, extraction procedures and chromatography were used as received from commercial suppliers without prior purification. During reaction workup, the reaction mixture was usually diluted to three times the original volume, and washed with equal volume of water and/or aqueous solutions as needed. All reagents were purchased from the Aldrich Chemical Company. 
(2R,3R,4S)-1-(tert-butyldiphenylsilyloxy)-3,4-(isopropylidenedioxy)-hex-5-yne-1,2-diol (4).

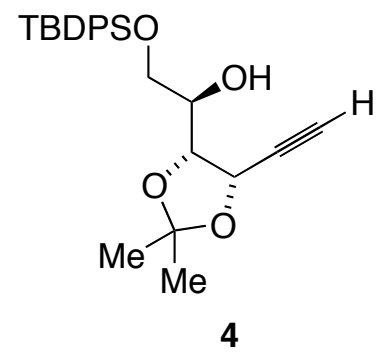

To a solution of $\mathrm{ClCH}_{2} \mathrm{PPh}_{3} \mathrm{Cl}(3.24 \mathrm{~g}, 9.32 \mathrm{mmol})$ in THF $(10 \mathrm{~mL})$ was added dropwise BuLi (1.6 $\mathrm{M}$ in hexanes, $6.8 \mathrm{ml}, 9.32 \mathrm{mmol})$, followed by TMEDA $(1.4 \mathrm{ml}, 9.32 \mathrm{mmol})$ and a solution of the 5-O-tert-butyldiphenylsilyl-2,3-O-isopropylidene-D-ribofuranose (3, $1.0 \mathrm{~g}, 2.33 \mathrm{mmol})$ in $10 \mathrm{~mL}$ of THF. The reaction was stirred at room temperature for 1.5 hours and quenched with a saturated solution of $\mathrm{NH}_{4} \mathrm{Cl}(130 \mathrm{~mL})$. The solution was extracted with $\mathrm{CH}_{2} \mathrm{Cl}_{2}$, dried with $\mathrm{MgSO}_{4}$ and evaporated. After a quick purification of the alkene this was dissolved in THF (5 $\mathrm{mL}$ ), cooled at $-78^{\circ} \mathrm{C}$ and treated dropwise with $n$-BuLi (1.6 $\mathrm{M}$ in hexanes, $5.7 \mathrm{~mL}, 9.10 \mathrm{mmol}$ ). After agitation during one hour the reaction was quenched with a saturated solution of $\mathrm{NH}_{4} \mathrm{Cl}$ and extracted with $\mathrm{CH}_{2} \mathrm{Cl}_{2}$. After purification $596 \mathrm{mg}(62 \%)$ of the alkynyl alcohol 4 was recovered.

m.p.: 82-83 ${ }^{\circ} \mathrm{C} ;[\alpha]^{23}{ }_{\mathrm{D}}=-18.88\left(\mathrm{CHCl}_{3}, \mathrm{c}=1.29\right)$; IR (neat): 3468, 3308, 3071, 3050, 2933, 2859, 2116, 1589, 1464, 1428, 1372, 1226, 1113, 1076, 967, 901, 865, 823, 740, $703 \mathrm{~cm}^{-1} ;{ }^{1} \mathrm{H}$ NMR (400 MHz, $\left.\mathrm{CDCl}_{3}\right) \delta$ 7.75-7.65 (m, 4H), 7.45-7.35 (m, 6H), $4.95(\mathrm{dd}, J=5.8,2.2 \mathrm{~Hz}, 1 \mathrm{H})$, $4.17(\mathrm{dd}, J=8.8,5.6 \mathrm{~Hz}, 1 \mathrm{H}), 4.08(\mathrm{~m}, 1 \mathrm{H}), 3.95(\mathrm{dd}, J=10.6,3.0 \mathrm{~Hz}, 1 \mathrm{H}), 3.90$ (dd, $J=10.4$, $4.4 \mathrm{~Hz}, 1 \mathrm{H}), 2.72(\mathrm{~d}, J=6.4 \mathrm{~Hz}, 1 \mathrm{H}), 2.57$ (d, $J=2.0 \mathrm{~Hz}, 1 \mathrm{H}), 1.51(\mathrm{~s}, 3 \mathrm{H}), 1.36(\mathrm{~s}, 3 \mathrm{H}), 1.10$ (s, 9H); ${ }^{13} \mathrm{C}$ NMR (100 MHz) $\delta 135.5,135.5,134.8,133.0,132.8,129.8,127.7,127.6,110.8,80.2$, $75.8,72.7,71.1,68.6,65.0,27.5,26.9,26.0,19.3$. 


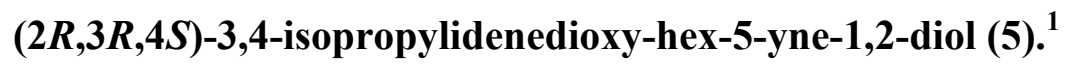

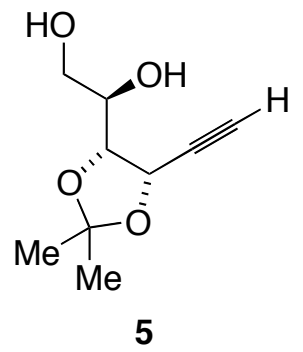

A solution of 5-O-tert-butyldiphenylsilyl-2,3-O-isopropylidene-D-ribofuranose $(3,1.00 \mathrm{~g}, 2.33$ $\mathrm{mmol})$ and $\mathrm{K}_{2} \mathrm{CO}_{3}(966 \mathrm{mg}, 6.99 \mathrm{mmol})$ in methanol $(6 \mathrm{~mL})$ at reflux was treated with a solution of dimethyl(diazo-2-oxopropyl)phosphonate $(1.34 \mathrm{~g}, 6.99 \mathrm{mmol})$ in methanol $(5 \mathrm{~mL})$ dropwise over 8 hours. The reaction mixture was cooled and the precipitate filtered. Then the solution was neutralized with $1 \mathrm{~N} \mathrm{HCl}$ and evaporated at reduced pressure. Extractive work-up (EtOAc / $\mathrm{H}_{2} \mathrm{O}$ ) and silica gel chromatography (hexanes : EtOAc 4:1 to 1:1) gave the alkynyl diol 5 (218 $\mathrm{mg}, 50 \%$ ).

m.p.: 71-72 ${ }^{\circ} \mathrm{C} ;[\alpha]^{23}{ }_{\mathrm{D}}=-16.91\left(\mathrm{CHCl}_{3}, \mathrm{c}=1.55\right)$; IR (neat): 3419, 3216, 2966, 2920, 2875, 2116, 1424, 1388, 1324, 1269, 1211, 1155, 1126, 1090, 1040, 1001, 923, 898, 834, 747, $724 \mathrm{~cm}^{-}$ ${ }^{1} ;{ }^{1} \mathrm{H}$ NMR $\left(600 \mathrm{MHz}, \mathrm{CDCl}_{3}\right) \delta 4.66(\mathrm{dd}, J=6.0,1.8 \mathrm{~Hz}, 1 \mathrm{H}), 4.08(\mathrm{t}, J=6.3 \mathrm{~Hz}, 1 \mathrm{H}), 3.81(\mathrm{~m}$, 1H), $3.74(\mathrm{dt}, J=12.0,3.6 \mathrm{~Hz}, 1 \mathrm{H}), 3.63(\mathrm{dd}, J=11.4,6.6 \mathrm{~Hz}, 1 \mathrm{H}), 3.11(\mathrm{bs}, 1 \mathrm{H}), 2.74(\mathrm{bs}, 1 \mathrm{H})$, $2.56(\mathrm{~d}, J=1.8 \mathrm{~Hz}, 1 \mathrm{H}), 1.46(\mathrm{~s}, 3 \mathrm{H}), 1.39(\mathrm{~s}, 3 \mathrm{H}) ;{ }^{13} \mathrm{C} \mathrm{NMR}(150 \mathrm{MHz}) \delta 110.8,81.6,81.5$, 74.7, 71.7, 66.7, 63.1, 26.7, 25.8; Anal. Calcd for $\mathrm{C}_{9} \mathrm{H}_{14} \mathrm{O}_{4}$ : C, 58.05; H, 7.58. Found: C, 57.90; $\mathrm{H}, 7.59$.

\footnotetext{
${ }^{1}$ Thiéry, J.-C.; Fréchou, C.; Demailly, G. Tetrahedron Lett. 2000, 41, 6337.
} 


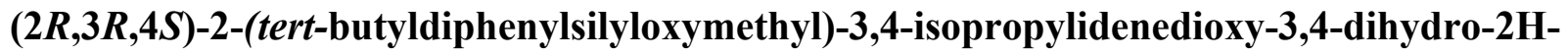
pyran (6) and (3S,4R,5R)-5-(tert-butyldiphenylsilyloxymethyl)-2-methylene-3,4isopropylidenedioxy-tetrahydrofuran (7).

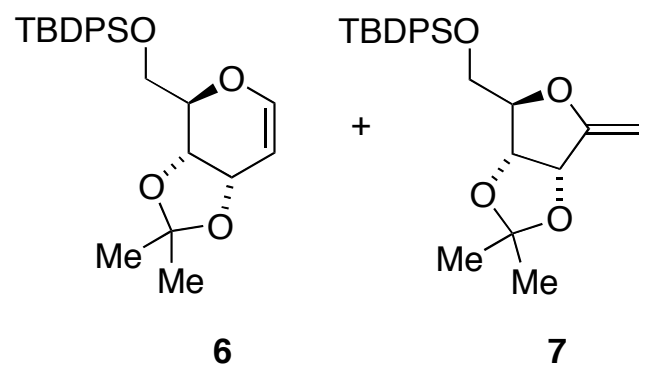

A flame-dried Schlenk flask fitted with a reflux condenser and a stir bar, under argon atmosphere, was charged with tungsten hexacarbonyl (68 mg, $0.19 \mathrm{mmol}$, dried under vacuum), alkynyl alcohol 4 (320 mg, $0.76 \mathrm{mmol}$, azeotropically dried from toluene), and DABCO (sublimed, $224 \mathrm{mg}, 1.95 \mathrm{mmol}$ ), and this mixture was dissolved in toluene $(3.6 \mathrm{~mL})$. The solution was deoxygenated and then irradiated under an inert atmosphere for $5 \mathrm{~h}$ at $350 \mathrm{~nm}$ (Rayonet photoreactor) without cooling, to reach approximately $70^{\circ} \mathrm{C}$. Volatile components were removed under reduced pressure and the product was purified by silica gel chromatography (hexanes : EtOAc 4:1 to $1: 1$ plus $0.1 \% \mathrm{Et}_{3} \mathrm{~N}$ ) to provide the 6 -endo glycal 6 (256 $\mathrm{mg}$, 80\% yield) contaminated by ca. $5 \%$ of the 5-exo glycal 7 .

\section{6-membered ring glycal (6):}

$[\alpha]^{23}{ }_{\mathrm{D}}=+84.44\left(\mathrm{CHCl}_{3}, \mathrm{c}=0.81\right)$; IR (neat): 2931, 2856, 1642, 1428, 1371, 1230, 1216, 1150, 1113, 1064,986, 867, 823, 789, 741, $702 \mathrm{~cm}^{-1} ;{ }^{1} \mathrm{H}$ NMR (400 MHz, $\left.\mathrm{CDCl}_{3}\right) \delta$ 7.73-7.70 (m, 4H), $7.45-7.35(\mathrm{~m}, 6 \mathrm{H}), 6.66(\mathrm{~d}, J=6.0 \mathrm{~Hz}, 1 \mathrm{H}), 5.10(\mathrm{dd}, J=5.8,4.6 \mathrm{~Hz}, 1 \mathrm{H}), 4.64(\mathrm{t}, J=5.0 \mathrm{~Hz}$, $1 \mathrm{H}), 4.11(\mathrm{dd}, J=9.8,5.4 \mathrm{~Hz}, 1 \mathrm{H}), 4.03(\mathrm{dd}, J=11.4,2.2 \mathrm{~Hz}, 1 \mathrm{H}), 3.93(\mathrm{dd}, J=11.6,4.8 \mathrm{~Hz}$, $1 \mathrm{H}), 3.49$ (ddd, $J=9.6,5.2,2.4 \mathrm{~Hz}, 1 \mathrm{H}), 1.40(\mathrm{~s}, 3 \mathrm{H}), 1.38(\mathrm{~s}, 3 \mathrm{H}), 1.07(\mathrm{~s}, 9 \mathrm{H}) ;{ }^{13} \mathrm{C}$ NMR $(100$ $\mathrm{MHz}) \delta 148.3,135.7,135.6,133.5,133.5,133.4,129.6,127.6,108.3,98.6,76.3,70.1,67.6$, 67.5, 62.8, 28.6, 26.8, 25.8, 19.3; Anal. Calcd for $\mathrm{C}_{25} \mathrm{H}_{32} \mathrm{O}_{4} \mathrm{Si}$ : C, 70.72; H, 7.60. Found: C, $70.78 ; \mathrm{H}, 7.63$.

\section{5-membered ring glycal (7):}

$[\alpha]^{23}=-25.84\left(\mathrm{CHCl}_{3}, \mathrm{c}=0.37\right)$; IR (neat): 2931, 2858, 1674, 1427, 1380, 1252, 1223, 1155 , 1113, 1083, 974, 870, 822, 741, $702 \mathrm{~cm}^{-1} ;{ }^{1} \mathrm{H}$ NMR (400 MHz, $\left.\mathrm{CDCl}_{3}\right) \delta$ 7.68-7.65 (m, 4H), 7.45-7.35 (m, 6H), $5.12(\mathrm{~d}, J=6.0 \mathrm{~Hz}, 1 \mathrm{H}), 4.78(\mathrm{~d}, J=6.0 \mathrm{~Hz}, 1 \mathrm{H}), 4.49(\mathrm{~s}, 1 \mathrm{H}), 4.41(\mathrm{~s}, 1 \mathrm{H})$, $4.22(\mathrm{~s}, 1 \mathrm{H}), 3.79(\mathrm{dd}, J=11.0,3.0 \mathrm{~Hz}, 1 \mathrm{H}), 3.71(\mathrm{dd}, J=11.4,2.6 \mathrm{~Hz}, 1 \mathrm{H}), 1.52$ (s, $3 \mathrm{H}), 1.40$ (s, 3H), $1.04(\mathrm{~s}, 9 \mathrm{H}) ;{ }^{13} \mathrm{C}$ NMR $(100 \mathrm{MHz}) \delta 163.3,135.6,135.5,132.9,132.6,129.9,129.8$, $127.8,127.7,112.6,85.9,84.0,80.8,80.3,64.8,27.1,26.7,25.9,19.0$. 
$(3 R, 4 R, 5 S)-4,5$-isopropylidenedioxy-2,3,4,5-tetrahydrooxepine-3-ol (8).

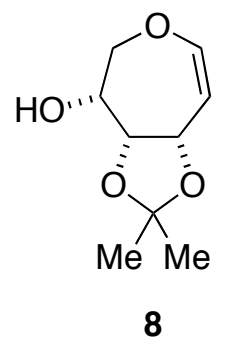

A flame-dried Schlenk flask fitted with a reflux condenser and a stir bar, under argon atmosphere, was charged with tungsten hexacarbonyl (58 mg, $0.16 \mathrm{mmol}$, dried under vacuum) and alkynyl diol 5 (200 mg, $1.07 \mathrm{mmol}$, azeotropically dried from toluene). This mixture was dissolved in freshly distilled dry THF $(5.4 \mathrm{~mL})$ and triethylamine $(1.35 \mathrm{~mL})$. The solution was deoxygenated and then irradiated under an inert atmosphere for $5 \mathrm{~h}$ at $350 \mathrm{~nm}$ (Rayonet photoreactor) without cooling, so that the solvent reflux point was reached. Volatile components were removed under reduced pressure and the product was purified by silica gel chromatography (hexanes : EtOAc 4:1 to $1: 1$ plus $\left.0.1 \% \mathrm{Et}_{3} \mathrm{~N}\right)$ gave the seven-membered glycal $8(136 \mathrm{mg}, 68 \%$ ) separated from approximately $2 \%$ of the six-membered glycal byproduct.

\section{7-membered ring glycal (8):}

$[\alpha]^{23}{ }_{\mathrm{D}}=-66.47\left(\mathrm{CHCl}_{3}, \mathrm{c}=0.73\right)$; IR (neat): 3472, 2985, 2926, 2855, 1640, 1459, 1372, 1332 , 1238, 1168, 1121, 1083, 1020, 979, 910, 863, 788, 734, $679 \mathrm{~cm}^{-1} ;{ }^{1} \mathrm{H}$ NMR $\left(400 \mathrm{MHz}, \mathrm{CDCl}_{3}\right) \delta$ $6.36(\mathrm{dd}, J=6.4,2.0 \mathrm{~Hz}, 1 \mathrm{H}), 5.16(\mathrm{dd}, J=6.4,2.0 \mathrm{~Hz}, 1 \mathrm{H}), 4.87(\mathrm{dt}, J=10.0,2.0 \mathrm{~Hz}, 1 \mathrm{H}), 4.39$ $(\mathrm{dt}, J=8.4,4.3 \mathrm{~Hz}, 1 \mathrm{H}), 4.22(\mathrm{dd}, J=12.4,5.2 \mathrm{~Hz}, 1 \mathrm{H}), 3.85$ (dd, $J=9.6,4.0 \mathrm{~Hz}, 1 \mathrm{H}), 3.75$ (dd, $J=12.4,8.0 \mathrm{~Hz}, 1 \mathrm{H}), 2.63(\mathrm{bs}, 1 \mathrm{H}), 1.46(\mathrm{~s}, 3 \mathrm{H}), 1.44(\mathrm{~s}, 3 \mathrm{H}) ;{ }^{13} \mathrm{C} \mathrm{NMR}(100 \mathrm{MHz}) \delta 148.6$, $109.5,108.8,80.1,72.7,71.9,65.9,27.2,26.5$.

\section{6-membered ring glycal:}

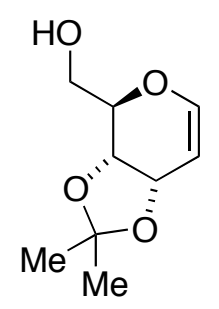

${ }^{1} \mathrm{H}$ NMR $\left(400 \mathrm{MHz}, \mathrm{CDCl}_{3}\right) \delta 6.64(\mathrm{~d}, J=6.0 \mathrm{~Hz}, 1 \mathrm{H}), 5.16(\mathrm{t}, J=5.2 \mathrm{~Hz}, 1 \mathrm{H}), 4.45(\mathrm{t}, J=5.0$ $\mathrm{Hz}, 1 \mathrm{H}), 4.03(\mathrm{dd}, J=10.0,5.6 \mathrm{~Hz}, 1 \mathrm{H}), 3.96(\mathrm{~m}, 1 \mathrm{H}), 3.83(\mathrm{dd}, J=11.8,5.0 \mathrm{~Hz}, 1 \mathrm{H}), 3.45$ $(\mathrm{ddd}, J=10.0,5.6,2.8 \mathrm{~Hz}, 1 \mathrm{H}), 1.47(\mathrm{~s}, 3 \mathrm{H}), 1.39$ (s, 3H); ${ }^{13} \mathrm{C} \mathrm{NMR}(100 \mathrm{MHz}) \delta 148.2,108.8$, $99.6,76.0,70.5,67.8,62.2,29.9,28.7$. 
$(3 R, 4 R, 5 S)$-3-acetoxy-4,5-isopropylidenedioxy-2,3,4,5-tetrahydrooxepine (9).

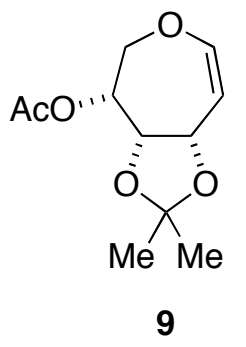

The same procedure for the synthesis of 8 was followed using alkynyl diol 5 (1.40 g, 7.53 $\mathrm{mmol}), \mathrm{W}(\mathrm{CO})_{6}(272 \mathrm{mg}, 0.753 \mathrm{mmol})$ and $\mathrm{Et}_{3} \mathrm{~N}(18.75 \mathrm{~mL})$ in THF $(75 \mathrm{~mL})$. In this case after the cycloisomerization reaction the crude was acetylated with $\mathrm{Ac}_{2} \mathrm{O}\left(1.9 \mathrm{~g}, 18.6 \mathrm{mmol}, \mathrm{Et}_{3} \mathrm{~N}\right.$ (1.8 mL), DMAP (20 mg) in $\mathrm{CH}_{2} \mathrm{Cl}_{2}(75 \mathrm{~mL})$, affording after silica chromatography (Hexanes : EtOAc 4:1 plus 0.1\% $\left.\mathrm{Et}_{3} \mathrm{~N}\right)$ the desired ester 9 (1.33 g, 82\%).

$[\alpha]^{23}=-77.89\left(\mathrm{CHCl}_{3}, \mathrm{c}=1.62\right)$; IR (neat): 3058, 2986, 2936, 2887, 1747, 1641, 1456, 1372, $1235,1171,1098,1066,1050,1026,990,942,897,867,805,748,715,660 \mathrm{~cm}^{-1} ;{ }^{1} \mathrm{H}$ NMR $(400$ $\left.\mathrm{MHz}, \mathrm{CDCl}_{3}\right) \delta 6.36(\mathrm{dd}, J=6.0,2.4 \mathrm{~Hz}, 1 \mathrm{H}), 5.48(\mathrm{dt}, J=6.4,3.6 \mathrm{~Hz}, 1 \mathrm{H}), 5.29(\mathrm{dd}, J=6.2$, $2.2 \mathrm{~Hz}, 1 \mathrm{H}), 4.89$ (dt, $J=9.4,2.2 \mathrm{~Hz}, 1 \mathrm{H}), 4.03$ (dd, $J=12.8,4.0 \mathrm{~Hz}, 1 \mathrm{H}), 3.89$ (dd, $J=12.8,6.2$ $\mathrm{Hz}, 1 \mathrm{H}), 3.82(\mathrm{dd}, J=9.6,3.6 \mathrm{~Hz}, 1 \mathrm{H}), 2.10$ (s, 3H), $1.41(\mathrm{~s}, 3 \mathrm{H}), 1.36(\mathrm{~s}, 3 \mathrm{H}) ;{ }^{13} \mathrm{C}$ NMR $(100$ MHz) $\delta 169.9,148.0,109.5,111.8,109.6$, 78.4, 72.1, 70.3, 67.5, 27.0, 26.2, 20.8; Anal. Calcd for $\mathrm{C}_{11} \mathrm{H}_{16} \mathrm{O}_{5}$ : C, 57.89; H, 7.07. Found: C, 57.85; H, 7.03.

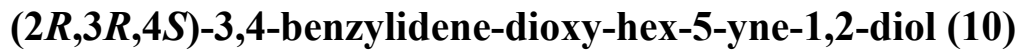

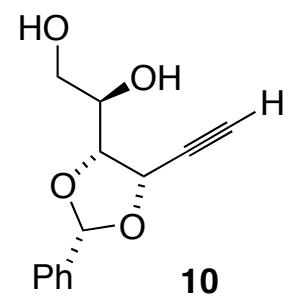

A solution of 2,3-O-(R)-benzylidene-D-ribofuranose ${ }^{2}(1.0663 \mathrm{~g}, 4.4778 \mathrm{mmol})$ and $\mathrm{K}_{2} \mathrm{CO}_{3}$ $(1.8527 \mathrm{~g}, 13.400 \mathrm{mmol})$ in methanol $(11.5 \mathrm{~mL})$ was heated to reflux. To this mixture was added a solution of dimethyl(diazo-2-oxopropyl)phosphonate $(2.5795 \mathrm{~g}, 13.434 \mathrm{mmol})$ in methanol $(9.5 \mathrm{~mL})$ dropwise over 8 hours. The reaction mixture was cooled and neutralized with $1 \mathrm{M} \mathrm{HCl}$ and evaporated at reduced pressure. Extractive work-up (EtOAc / $\left.\mathrm{H}_{2} \mathrm{O}\right)$ and silica gel

\footnotetext{
${ }^{2}$ Chan, L.; Just, G. Tetrahedron, 1990, 46, 151.
} 
chromatography (1:1 pentane/ethyl ether) gave the alkynyl diol $\mathbf{1 0}(0.3160 \mathrm{~g}, 30 \%)$ as a white solid.

m.p.: $126-127^{\circ} \mathrm{C} ;[\alpha]^{23}{ }_{\mathrm{D}}=-20.9(\mathrm{MeOH}, \mathrm{c}=0.88) ; \operatorname{IR}($ neat): 3291, 3172, 2926, 2119, 1732, 1466, 1406, 1314, 1270, 1221, 1088, 1069, 967, $759 \mathrm{~cm}^{-1} ;{ }^{1} \mathrm{H}$ NMR $\left(600 \mathrm{MHz}, \mathrm{CDCl}_{3}\right) \delta 7.49$ (mult, 2H); 7.41 (mult, 3H); 6.03 (s, 1H); 4.98 (dd, $J=4.8,2.0 \mathrm{~Hz}, 1 \mathrm{H}) ; 4.26(\mathrm{t}, J=5.6 \mathrm{~Hz}, 1 \mathrm{H})$; 3.92 (app dt, $J=5.6,3.9 \mathrm{~Hz}, 1 \mathrm{H}) ; 3.85(\mathrm{dd}, J=11.5,3.6 \mathrm{~Hz}, 1 \mathrm{H}) ; 3.76(\mathrm{dd}, J=11.8,5.6 \mathrm{~Hz}$, $1 \mathrm{H}) ; 2.64(\mathrm{~d}, J=2.2 \mathrm{~Hz}, 1 \mathrm{H}) ; 2.49(\mathrm{br} \mathrm{s}, 1 \mathrm{H}) ; 1.86(\mathrm{br} \mathrm{s}, 1 \mathrm{H}) ;{ }^{13} \mathrm{C} \mathrm{NMR}\left(150 \mathrm{MHz}, \mathrm{CD}_{3} \mathrm{OD}\right) \delta$ 137.9, 130.8, 128.2, 105.1, 84.6, 82.7, 76.6, 73.8, 69.3, 64.4; Anal. Calcd for $\mathrm{C}_{13} \mathrm{H}_{14} \mathrm{O}_{4}$ : C, 66.66; H, 6.02. Found: C, 66.43; H, 6.20.

\section{$(3 R, 4 R, 5 S)-3$-acetoxy-4,5-benzylidene-dioxy-2,3,4,5-tetrahydrooxepine (11)}

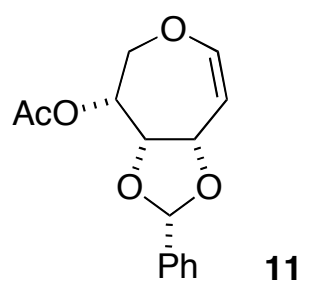

A flame-dried Schlenk flask, reflux condenser, and stir bar was charged with alkynyl diol $\mathbf{1 0}$ ( $0.1142 \mathrm{~g}, 0.4878 \mathrm{mmol}$, azeotropically dried from toluene), tungsten hexacarbonyl (0.0246 g, $0.0699 \mathrm{mmol})$ and, freshly sublimed DABCO $(0.1099 \mathrm{~g}, 0.9797 \mathrm{mmol})$. The mixture was dissolved in toluene $(2.5 \mathrm{~mL})$ and irradiated at $350 \mathrm{~nm}$ for $6 \mathrm{~h}$ at $70{ }^{\circ} \mathrm{C}$. Upon completion, volatile components were removed under reduced pressure. To the crude mixture was added $\mathrm{CH}_{2} \mathrm{Cl}_{2}(2.0 \mathrm{~mL})$, followed by acetic anhydride $(70 \mu \mathrm{L}, 0.742 \mathrm{mmol})$, and DMAP $(0.1197 \mathrm{~g}$, $0.9798 \mathrm{mmol}$ ). After $1 \mathrm{~h}$, volatiles were removed and the product was purified by silica gel chromatography (9:1 pentane/ethyl ether plus $1 \%$ triethylamine) to give the seven-membered glycal $11(0.1102 \mathrm{~g}, 82 \%)$ as a clear oil.

$[\alpha]^{23}=-9.9\left(\mathrm{CHCl}_{3}, \mathrm{c}=0.74\right)$; IR(neat): 3635, 3474, 3061, 3034, 2918, 2892, 1744, 1638, 1494, 1458, 1371, 1324, 1233, 1104, 1069, $969 \mathrm{~cm}^{-1} ;{ }^{1} \mathrm{H}$ NMR (600 MHz, CDCl $) \delta 7.47$ (mult, 2H); 7.38 (mult, 3H); 6.43 (dd, $J=6.2,2.4 \mathrm{~Hz}, 1 \mathrm{H}$ ); 6.16 (s, 1H); 5.69 (app dt, $J=6.2,3.8 \mathrm{~Hz}, 1 \mathrm{H}$ ); $5.42(\mathrm{dd}, J=6.2,2.4 \mathrm{~Hz}, 1 \mathrm{H}) ; 4.97(\mathrm{dt}, J=10.0,2.4,1.9 \mathrm{~Hz}, 1 \mathrm{H}) ; 4.15(\mathrm{dd}, J=12.9,3.8 \mathrm{~Hz}$, $1 \mathrm{H}) ; 4.06(\mathrm{dd}, J=9.5,3.3 \mathrm{~Hz}, 1 \mathrm{H}) ; 3.95(\mathrm{dd}, 12.9,6.7 \mathrm{~Hz}, 1 \mathrm{H}) ; 2.05(\mathrm{~s}, 3 \mathrm{H}) ;{ }^{13} \mathrm{C}$ NMR $(150$ $\left.\mathrm{MHz}, \mathrm{CDCl}_{3}\right) \delta 170.3,148.5,139.3,129.3,128.5,126.3,111.8,104.4,80.3,72.2,70.7,67.6$, 21.1; Anal. Calcd for $\mathrm{C}_{15} \mathrm{H}_{16} \mathrm{O}_{5}$ : C, 65.21; H, 5.84. Found: C, 65.42; H, 5.99. 
nOe spectrum of $11\left(600 \mathrm{MHz}, \mathrm{CDCl}_{3}\right)$ :

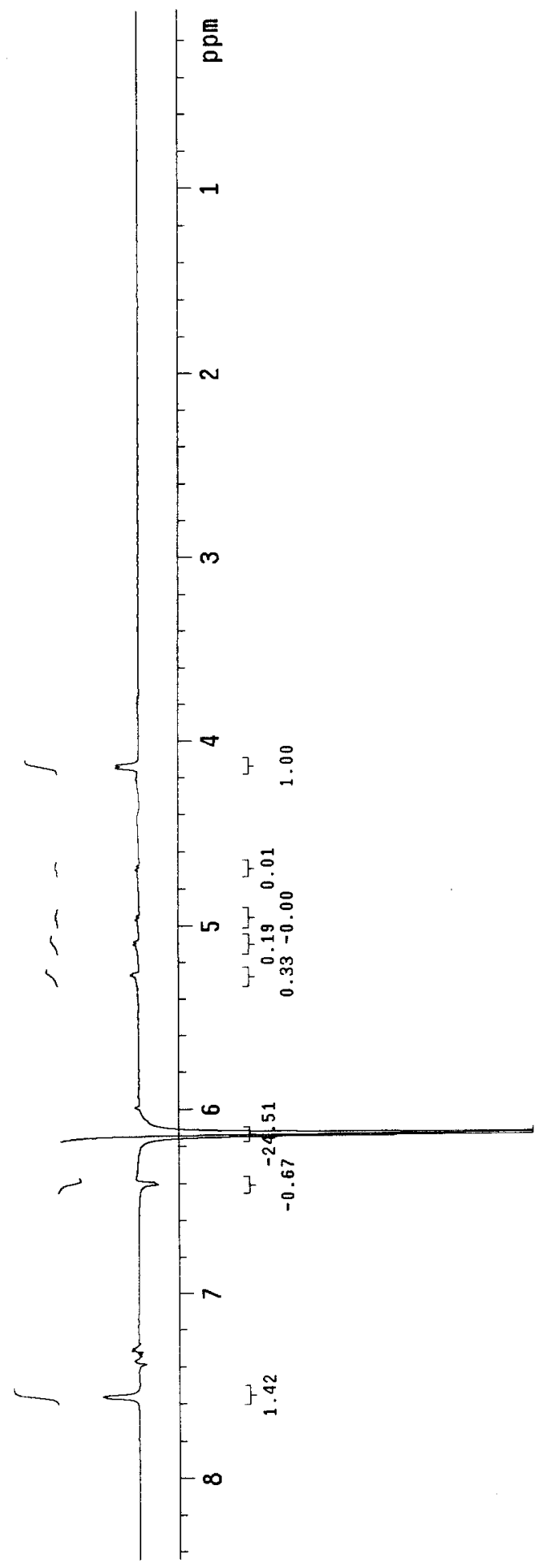




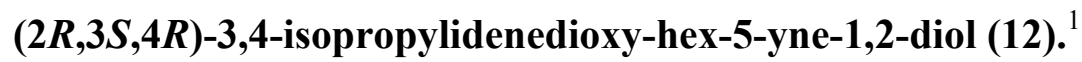

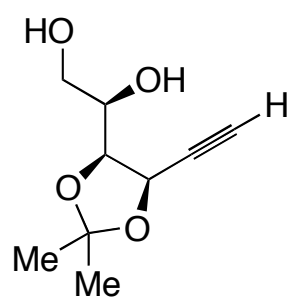

12

The procedure for the synthesis of 5 was followed, using 2,3-O-isopropylidene-D-lyxofuranose (583 mg, $3.07 \mathrm{mmol}), \mathrm{K}_{2} \mathrm{CO}_{3}(1.27 \mathrm{~g}, 9.21 \mathrm{mmol})$ in methanol $(8 \mathrm{~mL})$, and the solution of dimethyl(diazo-2-oxopropyl)phosphonate $(1.90 \mathrm{~g}, 9.21 \mathrm{mmol})$ in methanol $(6.7 \mathrm{~mL})$. After chromatographic purification, the alkynyl diol 12 (284 mg, 49\% yield) was obtained.

$[\alpha]^{23}=-21.64\left(\mathrm{CHCl}_{3}, \mathrm{c}=1.65\right)$; IR (neat): 3401, 3291, 2989, 2937, 2896, 2122, 1382, 1242 , 1214, 1162, 1130, 1064, 879, 808, $641 \mathrm{~cm}^{-1} ;{ }^{1} \mathrm{H}$ NMR (600 MHz, $\left.\mathrm{CDCl}_{3}\right) \delta 4.61(\mathrm{dd}, J=7.5,2.1$ Hz, 1H), 4.07 (dd, $J=8.1,2.7$ Hz, 1H), 3.73 (m, 3H), 3.07 (d, $J=6.6 \mathrm{~Hz}, 1 \mathrm{H}), 2.89$ (bs, 1H), $2.55(\mathrm{~d}, J=1.8 \mathrm{~Hz}, 1 \mathrm{H}), 1.46(\mathrm{~s}, 3 \mathrm{H}), 1.41(\mathrm{~s}, 3 \mathrm{H}) ;{ }^{13} \mathrm{C} \mathrm{NMR}(150 \mathrm{MHz}) \delta 111.0,82.2,80.2$, $75.1,69.7,66.7,64.3,26.5,26.0$.

\section{(3R,4S,5R)-4,5-isopropylidenedioxy-2,3,4,5-tetrahydrooxepine-3-ol (13-OH).}

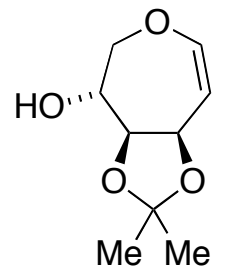

The same procedure for the synthesis of $\mathbf{8}$ was followed using alkynyl diol $12(211 \mathrm{mg}, 1.14$ $\mathrm{mmol}), \mathrm{W}(\mathrm{CO})_{6}(52 \mathrm{mg}, 0.114 \mathrm{mmol})$ and $\mathrm{Et}_{3} \mathrm{~N}(3.5 \mathrm{~mL})$ in THF (14.2 mL). After silica chromatography (Hexanes : EtOAc $4: 1$ to $1: 1$ plus $\left.0.1 \% \mathrm{Et}_{3} \mathrm{~N}\right)$ the 7 -endo glycal $(157 \mathrm{mg}, 75 \%$ yield) was obtained.

m.p.: $129-130{ }^{\circ} \mathrm{C} ;[\alpha]^{23}=-10.65\left(\mathrm{CHCl}_{3}, \mathrm{c}=1.11\right)$; IR (neat): 3431, 2978, 2928, 2874, 1642, $1448,1422,1374,1295,1268,1252,1233,1168,1143,1100,1083,1058,1030,998,983,915$, 863, 835, 793, 746, 705, $650 \mathrm{~cm}^{-1} ;{ }^{1} \mathrm{H} \mathrm{NMR}\left(400 \mathrm{MHz}, \mathrm{CDCl}_{3}\right) \delta 6.37$ (dd, $\left.J=6.6,1.8 \mathrm{~Hz}, 1 \mathrm{H}\right)$, $5.08(\mathrm{dd}, J=6.6,1.4 \mathrm{~Hz}, 1 \mathrm{H}), 4.54(\mathrm{~d}, J=9.6 \mathrm{~Hz}, 1 \mathrm{H}), 4.06(\mathrm{~m}, 2 \mathrm{H}), 3.99$ (d, $J=13.6,3.6 \mathrm{~Hz}$, $1 \mathrm{H}), 3.92$ (dd, $J=9.6,7.2 \mathrm{~Hz}, 1 \mathrm{H}), 2.62$ (bs, 1H), 1.46 (s, 3H), 1.44 (s, 3H); ${ }^{13} \mathrm{C}$ NMR $(100$ $\mathrm{MHz}) \delta 149.0,109.8,107.0,83.8,75.1,73.4,73.3,27.0,27.0$; Anal. Calcd for $\mathrm{C}_{9} \mathrm{H}_{14} \mathrm{O}_{4}$ : C, 58.05; H, 7.58. Found: C, 57.97; H, 7.35. 
(3R,4S,5R)-3-acetoxy-4,5-isopropylidenedioxy-2,3,4,5-tetrahydrooxepine (13).

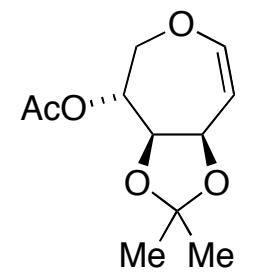

13

Following the same sequence as with 8, alkynyl diol $12(211 \mathrm{mg}, 1.14 \mathrm{mmol}), \mathrm{W}(\mathrm{CO})_{6}(52 \mathrm{mg}$, $0.114 \mathrm{mmol})$ and $\mathrm{Et}_{3} \mathrm{~N}(3.5 \mathrm{~mL})$ in THF $(14.2 \mathrm{~mL})$ were irradiated. After 3 hours the crude was acetylated with $\mathrm{Ac}_{2} \mathrm{O}(176 \mathrm{mg}, 1.72 \mathrm{mmol})$ and DMAP (401 mg, $\left.3.42 \mathrm{mmol}\right)$. After purification the desired acetate 13 (199 mg, 81\% yield) was obtained.

$[\alpha]^{23}=-65.65\left(\mathrm{CHCl}_{3}, \mathrm{c}=0.63\right)$; IR (neat): 2987, 2932, 2851, 1743, 1643, 1452, 1372, 1230, 1172, 1133, 1085, 1062, 1019, 988, 933, 867, 842, 803, 742, 705, $660 \mathrm{~cm}^{-1} ;{ }^{1} \mathrm{H}$ NMR (400 MHz, $\left.\mathrm{CDCl}_{3}\right) \delta 6.38(\mathrm{dd}, J=6.2,1.8 \mathrm{~Hz}, 1 \mathrm{H}), 5.15(\mathrm{dd}, J=7.7,2.0 \mathrm{~Hz}, 1 \mathrm{H}), 5.05(\mathrm{dd}, J=6.2,1.4 \mathrm{~Hz}$, $1 \mathrm{H}), 4.64(\mathrm{dt}, J=9.6,1.6 \mathrm{~Hz}, 1 \mathrm{H}), 4.23(\mathrm{dd}, J=14.0,1.2 \mathrm{~Hz}, 1 \mathrm{H}), 4.18(\mathrm{dd}, J=10.0,7.6 \mathrm{~Hz}$, $1 \mathrm{H}), 4.01(\mathrm{dd}, J=14.0,2.8 \mathrm{~Hz}, 1 \mathrm{H}), 2.15(\mathrm{~s}, 3 \mathrm{H}), 1.45(\mathrm{~s}, 3 \mathrm{H}), 1.43(\mathrm{~s}, 3 \mathrm{H}) ;{ }^{13} \mathrm{C}$ NMR $(100$ $\mathrm{MHz}) \delta 170.6,149.1,119.8,109.9,80.2,75.6,73.5,68.7,27.0,26.9,20.7$; Anal. Calcd for $\mathrm{C}_{11} \mathrm{H}_{16} \mathrm{O}_{5}$ : C, 57.89; H, 7.07. Found: C, 57.82; H, 7.12.

(2R,3S,4S)-3,4-isopropylidenedioxy-hex-5-yne-1,2-diol (14). ${ }^{1}$

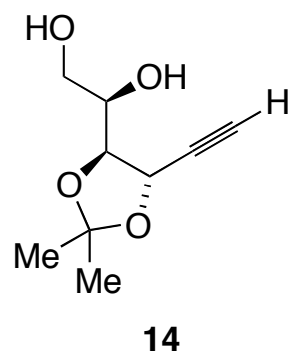

The same procedure for the synthesis of 5 was followed using 1-O-benzoate-2,3-Oisopropylidene-D-xylopyranose $(798 \mathrm{mg}, 2.71 \mathrm{mmol}), \mathrm{K}_{2} \mathrm{CO}_{3}(1.12 \mathrm{~g}, 8.13 \mathrm{mmol})$ in methanol $(6.8 \mathrm{~mL})$, and the solution of dimethyl(diazo-2-oxopropyl)phosphonate $(1.55 \mathrm{~g}, 8.13 \mathrm{mmol})$ in methanol (5.8 mL). After chromatographic purification, the alkynyl diol 14 (276 mg, 55\% yield) was obtained.

$[\alpha]^{23}=-20.27\left(\mathrm{CHCl}_{3}, \mathrm{c}=1.18\right)$; IR (neat): 3429, 3291, 2989, 2936, 2896, 2115, 1710, 1640, 1456. 1382, 1243, 1215, 1162, 1129, 1060, 879, 809, $658 \mathrm{~cm}^{-1} ;{ }^{1} \mathrm{H}$ NMR $\left(600 \mathrm{MHz}, \mathrm{CDCl}_{3}\right) \delta$ 
4.62 (dd, $J=7.8,1.8 \mathrm{~Hz}, 1 \mathrm{H}), 4.09$ (dd, $J=7.8,3.0 \mathrm{~Hz}, 1 \mathrm{H}), 3.74$ (m, 3H), 2.86 (bs, 2H), 2.55 (d, $J=1.8 \mathrm{~Hz}, 1 \mathrm{H}), 1.47$ (s, 3H), $1.42(\mathrm{~s}, 3 \mathrm{H}) ;{ }^{13} \mathrm{C} \mathrm{NMR}(150 \mathrm{MHz}) \delta 111.0,82.3,80.2,75.1$, $69.6,66.7,64.4,26.5,26.0$.

$(3 R, 4 S, 5 S)-4,5$-isopropylidenedioxy-2,3,4,5-tetrahydrooxepine-3-ol (15-OH).

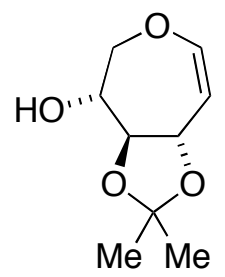

The same procedure for the synthesis of $\mathbf{8}$ was followed using alkynyl diol 14 (207 mg, 1.11 $\mathrm{mmol}), \mathrm{W}(\mathrm{CO})_{6}(63 \mathrm{mg}, 0.16 \mathrm{mmol})$ and $\mathrm{Et}_{3} \mathrm{~N}(2.6 \mathrm{~mL})$ in THF $(5.3 \mathrm{~mL})$. After silica chromatography (Hexanes : EtOAc 4:1 to 1:1 plus $0.1 \% \mathrm{Et}_{3} \mathrm{~N}$ ), the 7-endo glycal (92 $\mathrm{mg}, 45 \%$ yield) was isolated.

$[\alpha]^{23}=-8.67\left(\mathrm{CHCl}_{3}, \mathrm{c}=0.98\right)$; IR (neat): 3434, 2980, 2946, 2876, 1644, 1449, 1422, 1376, 1296, 1268, 1253, 1235, 1169, 1144, 1100, 1059, 1031, 984, 916, 863, 836, 795, 747, $706 \mathrm{~cm}^{-1}$; ${ }^{1} \mathrm{H}$ NMR (400 MHz, $\left.\mathrm{CDCl}_{3}\right) \delta 6.35(\mathrm{dd}, J=6.4,2.0 \mathrm{~Hz}, 1 \mathrm{H}), 5.05(\mathrm{dd}, J=6.4,1.6 \mathrm{~Hz}, 1 \mathrm{H}), 4.52$ (dt, $J=9.8,1.8 \mathrm{~Hz}, 1 \mathrm{H}), 3.98(\mathrm{~m}, 3 \mathrm{H}), 3.90$ (dd, $J=9.8,7.4 \mathrm{~Hz}, 1 \mathrm{H}), 3.13$ (bs, $1 \mathrm{H}), 1.41(\mathrm{~s}, 3 \mathrm{H})$, 1.40 (s, 3H); ${ }^{13} \mathrm{C}$ NMR (100 MHz) $\delta$ 149.0, 109.7, 106.8, 83.7, 75.2, 73.3, 73.0, 26.9, 26.9; Anal. Calcd for $\mathrm{C}_{9} \mathrm{H}_{14} \mathrm{O}_{4}: \mathrm{C}, 58.05 ; \mathrm{H}, 7.58$. Found: C, 57.97; H, 7.57.

(3R,4S,5S)-3-acetoxy-4,5-isopropylidenedioxy-2,3,4,5-tetrahydrooxepine (15).

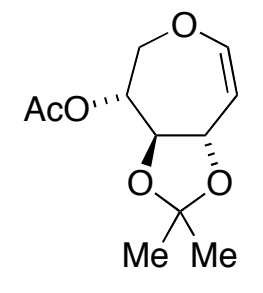

15

Following the same sequence as with 9, alkynyl diol 14 (253 mg, $1.36 \mathrm{mmol}), \mathrm{W}(\mathrm{CO})_{6}(49 \mathrm{mg}$, $0.136 \mathrm{mmol})$ and $\mathrm{Et}_{3} \mathrm{~N}(3.4 \mathrm{~mL})$ in THF $(13.6 \mathrm{~mL})$ were irradiated. After 3 hours the crude was acetylated with $\mathrm{Ac}_{2} \mathrm{O}(211 \mathrm{mg}, 2.06 \mathrm{mmol})$ and DMAP (481 mg, $\left.4.08 \mathrm{mmol}\right)$. After purification the desired acetate $\mathbf{1 5}$ (179 $\mathrm{mg}, 61 \%)$ was obtained. 
$[\alpha]^{23}{ }_{\mathrm{D}}=-65.25\left(\mathrm{CHCl}_{3}, \mathrm{c}=0.36\right)$; IR (neat): 2986, 2935, 2850, 1741, 1643, 1450, 1372, 1230, 1172, 1132, 1085, 1061, 1018, 988, 932, 867, 842, 802, 789, 741, $704 \mathrm{~cm}^{-1}$; ${ }^{1} \mathrm{H}$ NMR (400 MHz, $\left.\mathrm{CDCl}_{3}\right) \delta 6.38(\mathrm{dd}, J=6.4,2.0 \mathrm{~Hz}, 1 \mathrm{H}), 5.15(\mathrm{dd}, J=7.8,1.8 \mathrm{~Hz}, 1 \mathrm{H}), 5.05(\mathrm{dd}, J=6.6,1.40$ $\mathrm{Hz}, 1 \mathrm{H}), 4.65$ (dt, $J=10.0,1.6 \mathrm{~Hz}, 1 \mathrm{H}), 4.23(\mathrm{dd}, J=14.0,1.2 \mathrm{~Hz}, 1 \mathrm{H}), 4.18$ (dd, $J=9.6,7.6$ $\mathrm{Hz}, 1 \mathrm{H}), 4.01$ (dd, $J=14.4,2.8 \mathrm{~Hz}, 1 \mathrm{H}), 2.16$ (s, 3H), 1.45 (s, 3H), 1.43 (s, 3H); ${ }^{13} \mathrm{C}$ NMR $(100$ MHz) $\delta 171.2,149.2,109.8,105.8,80.3,75.6,73.5,73.2,27.0,26.9,21.2$; Anal. Calcd for $\mathrm{C}_{11} \mathrm{H}_{16} \mathrm{O}_{5}$ : C, 57.89; H, 7.07. Found: C, 57.91; H, 7.17.

\section{2,3-O-isopropylidene-4-O-p-nitrobenzoate- $\alpha, \beta$-L-arabino-benzoate-pyranoside (A).}

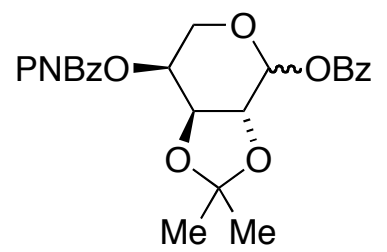

A flame dried flask was charged with 1-O-benzoate-2,3-O-isopropylidene-D-xylopyranose (1.0 g, $3.40 \mathrm{mmol}), \mathrm{PPh}_{3}(1.77 \mathrm{~g}, 6.80 \mathrm{mmol}), p$-nitrobenzoic acid $(1.14 \mathrm{~g}, 6.80 \mathrm{mmol})$ and anhydrous ether $(160 \mathrm{~mL})$. This mixture was cooled at $0^{\circ} \mathrm{C}$ and then DEAD $(40 \%$ in toluene, $3.1 \mathrm{~mL}, 6.80$ mmol) was added dropwise. After removal of the cooling bath the reaction was stirred overnight. Once the reaction was done the solvent was evaporated and the residue was chromatographed (Hexanes : EtOAc 4:1) providing the $p$-nitrobenzoate L-arabinose product $\mathbf{A}$ $(1.18 \mathrm{~g}, 78 \%)$.

${ }^{1} \mathrm{H}$ NMR (400 MHz, $\left.\mathrm{CDCl}_{3}\right) \delta 8.32(\mathrm{~m}, 2 \mathrm{H}), 8.25(\mathrm{~m}, 2 \mathrm{H}), 8.12(\mathrm{~m}, 2 \mathrm{H}), 7.59(\mathrm{~m}, 1 \mathrm{H}), 7.45(\mathrm{~m}$, 2H), 6.09 (d, $J=8.0 \mathrm{~Hz}, 1 \mathrm{H}), 5.68(\mathrm{~d}, J=2.4 \mathrm{~Hz}, 1 \mathrm{H}), 4.30$ (d, $J=12.8 \mathrm{~Hz}, 1 \mathrm{H}), 4.18$ (dd, $J=$ 9.6, 8.4 Hz, 1H), 3.99 (dd, $J=13.6,2.0 \mathrm{~Hz}, 1 \mathrm{H}), 3.92$ (dd, $J=9.8,3.0 \mathrm{~Hz}, 1 \mathrm{H}), 1.49$ (s, $3 \mathrm{H})$, $1.44(\mathrm{~s}, 3 \mathrm{H}) ;{ }^{13} \mathrm{C}$ NMR $(150 \mathrm{MHz}) \delta 164.8,163.9,150.8,134.8,133.8,130.9,130.2,128.7$, $128.4,123.7,111.7,94.5,76.5,72.7,69.5,66.8,26.5,26.4$. 


\section{(2S,3S,4S)-3,4-isopropylidenedioxy-hex-5-yne-1,2-diol (16).}

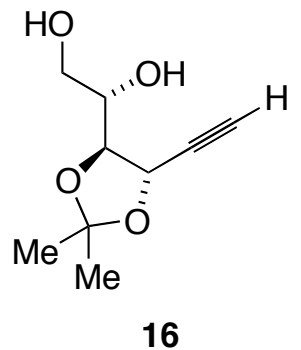

The procedure for the synthesis of $\mathbf{5}$ was followed, using protected L-arabinose derivative $\mathbf{A}$ (prepared above, $1.41 \mathrm{~g}, 3.17 \mathrm{mmol}), \mathrm{K}_{2} \mathrm{CO}_{3}(1.96 \mathrm{~g}, 14.26 \mathrm{mmol})$ in methanol $(8 \mathrm{~mL})$, and the solution of dimethyl(diazo-2-oxopropyl)phosphonate $(2.71 \mathrm{~g}, 14.26 \mathrm{mmol})$ in methanol $(6.7$ $\mathrm{mL})$. After chromatography purification the alkynyl diol 16 (64.8 mg, 11\%) was obtained.

${ }^{1} \mathrm{H}$ NMR $\left(400 \mathrm{MHz}, \mathrm{CDCl}_{3}\right) \delta 4.67(\mathrm{dd}, J=6.6,1.8 \mathrm{~Hz}, 1 \mathrm{H}), 4.11(\mathrm{dd}, J=6.6,5.4 \mathrm{~Hz}, 1 \mathrm{H}), 3.85$ (m, 1H), $3.74(\mathrm{~m}, 1 \mathrm{H}), 3.66(\mathrm{dd}, J=11.4,6.2 \mathrm{~Hz}, 1 \mathrm{H}), 3.06$ (bs, 1H), 2.69 (bs, 1H), 2.55 (d, $J=$ $2.0 \mathrm{~Hz}, 1 \mathrm{H}), 1.48(\mathrm{~s}, 3 \mathrm{H}), 1.40(\mathrm{~s}, 3 \mathrm{H}) ;{ }^{13} \mathrm{C} \mathrm{NMR}(150 \mathrm{MHz}) \delta 110.8,81.8,81.5,74.7,71.5$, $66.5,63.1,26.8,25.8$.

\section{(3S,4S,5S)-4,5-isopropylidenedioxy-2,3,4,5-tetrahydrooxepine-3-ol (17-OH).}

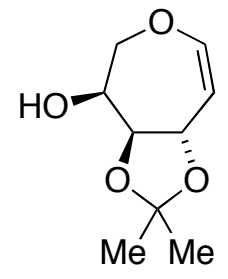

The same procedure for the synthesis of $\mathbf{8}$ was followed using alkynyl diol 16 (64.8 $\mathrm{mg}, 0.35$ $\mathrm{mmol}), \mathrm{W}(\mathrm{CO})_{6}(19 \mathrm{mg}, 0.05 \mathrm{mmol})$ and $\mathrm{Et}_{3} \mathrm{~N}(425 \mu \mathrm{L})$ in THF $(1.7 \mathrm{~mL})$. In the crude NMR the product was observed, but could not be isolated as the free alcohol after silica chromatography (Hexanes : EtOAc 4:1 to $1: 1$ plus $0.1 \% \mathrm{Et}_{3} \mathrm{~N}$ ).

${ }^{1} \mathrm{H}$ NMR $\left(400 \mathrm{MHz}, \mathrm{CDCl}_{3}\right) \delta 6.36(\mathrm{dd}, J=6.2,2.2 \mathrm{~Hz}, 1 \mathrm{H}), 5.16(\mathrm{dd}, J=6.2,1.8 \mathrm{~Hz}, 1 \mathrm{H}), 4.87$ (app. d, $J=9.6 \mathrm{~Hz}, 1 \mathrm{H}), 4.38(\mathrm{dt}, J=8.3,4.2 \mathrm{~Hz}, 1 \mathrm{H}), 4.20(\mathrm{dd}, J=12.4,5.2 \mathrm{~Hz}, 1 \mathrm{H}), 3.84$ (dd, $J=9.6,4.0 \mathrm{~Hz}, 1 \mathrm{H}), 3.75(\mathrm{~m}, 1 \mathrm{H}), 3.01(\mathrm{bs}, 1 \mathrm{H}), 1.45$ (s, 3H), 1.43 (s, 3H). 
$(3 S, 4 S, 5 S)-3$-acetoxy-4,5-isopropylidenedioxy-2,3,4,5-tetrahydrooxepine (17).

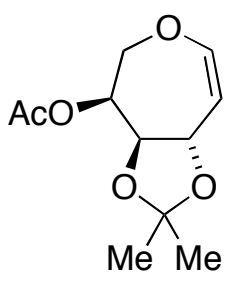

17

Following the same sequence as with 9, alkynyl diol $16(38.4 \mathrm{mg}, 0.21 \mathrm{mmol}), \mathrm{W}(\mathrm{CO})_{6}(11 \mathrm{mg}$, $0.03 \mathrm{mmol})$ and $\mathrm{Et}_{3} \mathrm{~N}(500 \mu \mathrm{L})$ in THF $(2.0 \mathrm{~mL})$ were irradiated. After 4 hours the crude was acetylated with $\mathrm{Ac}_{2} \mathrm{O}(32 \mathrm{mg}, 0.31 \mathrm{mmol})$ and DMAP (73 mg, $\left.0.63 \mathrm{mmol}\right)$. After purification the desired acetate 17 (22.5 $\mathrm{mg}, 63 \%$ yield) was obtained.

$[\alpha]^{23}=+52.86\left(\mathrm{CHCl}_{3}, \mathrm{c}=0.69\right)$; IR (neat): 2986, 2930, 2857, 1747, 1640, 1456, 1371, 1233, 1171, 1098, 1066, 1050, 1027, 990, 979, 942, 890, 866, 804, 747, $714 \mathrm{~cm}^{-1}$; ${ }^{1} \mathrm{H}$ NMR (400 MHz, $\left.\mathrm{CDCl}_{3}\right) \delta 6.39(\mathrm{dd}, J=6.4,2.4 \mathrm{~Hz}, 1 \mathrm{H}), 5.51(\mathrm{dt}, J=6.4,3.6 \mathrm{~Hz}, 1 \mathrm{H}), 5.32(\mathrm{dd}, J=6.0,2.0 \mathrm{~Hz}$, $1 \mathrm{H}), 4.92(\mathrm{dt}, J=9.6,2.2 \mathrm{~Hz}, 1 \mathrm{H}), 4.06(\mathrm{dd}, J=13.0,4.2 \mathrm{~Hz}, 1 \mathrm{H}), 3.93(\mathrm{dd}, J=13.2,6.0 \mathrm{~Hz}$, $1 \mathrm{H}), 3.85(\mathrm{dd}, J=9.6,3.6 \mathrm{~Hz}, 1 \mathrm{H}), 2.13(\mathrm{~s}, 3 \mathrm{H}), 1.44(\mathrm{~s}, 3 \mathrm{H}), 1.40(\mathrm{~s}, 3 \mathrm{H}) ;{ }^{13} \mathrm{C} \mathrm{NMR}(100 \mathrm{MHz})$ ठ $170.0,148.1,111.9,109.8,78.5,72.2,70.4,67.6,27.0,26.3,20.9$.

\section{(3S,4R,5S,6S)-3,4-isopropylidenedioxy-hept-1-yne-5,6-diol (18)}

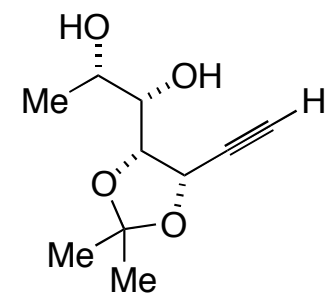

18

A solution of 2,3-O-isopropylidene-L-rhamnose $(0.5395 \mathrm{~g}, 2.642 \mathrm{mmol})$ and $\mathrm{K}_{2} \mathrm{CO}_{3}(1.0960 \mathrm{~g}$, $7.9271 \mathrm{mmol})$ in methanol $(6.7 \mathrm{~mL})$ was heated to reflux. To this solution was added a solution of dimethyl(diazo-2-oxopropyl)phosphonate (1.5222 g, $7.9273 \mathrm{mmol})$ in methanol (5.6 $\mathrm{mL})$ dropwise over 8 hours. The reaction mixture was cooled and neutralized with $1 \mathrm{M} \mathrm{HCl}$ and evaporated at reduced pressure. Extractive work-up (EtOAc / $\mathrm{H}_{2} \mathrm{O}$ ) and silica gel chromatography (1:1 pentane/ethyl ether) gave the alkynyl diol $18(0.2094 \mathrm{~g}, 40 \%)$ as a clear oil. $[\alpha]^{23}=+33.0\left(\mathrm{CHCl}_{3}, \mathrm{c}=2.10\right)$; IR(neat): 3435, 3294, 2988, 2934, 2122, 1726, 1636, 1456, 1380, 1243, 1216, 1164, 1052, $879 \mathrm{~cm}^{-1} ;{ }^{1} \mathrm{H}$ NMR (400 MHz, $\left.\mathrm{CDCl}_{3}\right) \delta 4.71(\mathrm{dd}, J=7.8,2.0$ $\mathrm{Hz}, 1 \mathrm{H}), 4.27$ (dd, $J=7.6,2.5 \mathrm{~Hz}, 1 \mathrm{H}), 3.89$ (mult, $1 \mathrm{H}), 3.51$ (dd, $J=5.4,2.5 \mathrm{~Hz}, 1 \mathrm{H}), 2.55$ (d, $J$ 
$=1.9 \mathrm{~Hz}, 1 \mathrm{H}), 2.32(\mathrm{br} \mathrm{s}, 2 \mathrm{H}), 1.50(\mathrm{~s}, 3 \mathrm{H}), 1.44(\mathrm{~s}, 3 \mathrm{H}), 1.32(\mathrm{~d}, J=6.4 \mathrm{~Hz}, 3 \mathrm{H}) ;{ }^{13} \mathrm{C}$ NMR $(100$ $\left.\mathrm{MHz}, \mathrm{CDCl}_{3}\right) \delta 111.1,81.1,80.5,75.3,71.8,69.7,67.1,26.8,26,4,19.8$; Anal. Calcd for $\mathrm{C}_{10} \mathrm{H}_{16} \mathrm{O}_{4}$ : C, 59.98; H, 8.05. Found: C, 60.09; H, 8.11.

\section{$(2 S, 3 S, 4 S, 5 S)-3$-acetoxy-4,5-isopropylidenedioxy-2-methyl-2,3,4,5-tetrahydrooxepine (19)}

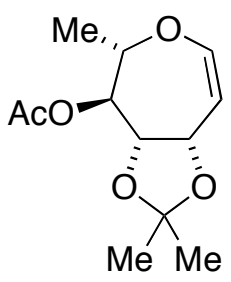

19

Method A (with $\mathrm{Et}_{3} \mathrm{~N}$ as base and THF as solvent): A flame-dried Schlenk flask, reflux condenser, and stir bar was charged with alkynyl diol 18 (130.1 mg, $0.6499 \mathrm{mmol}$, azeotropically dried from toluene) and tungsten hexacarbonyl (34.5 mg, $0.0980 \mathrm{mmol})$. Anhydrous THF (6.0 $\mathrm{mL})$ and $\mathrm{Et}_{3} \mathrm{~N}(1.5 \mathrm{~mL})$ were added and the reaction was irradiated at $350 \mathrm{~nm}$ for $6 \mathrm{~h}$ at $65^{\circ} \mathrm{C}$ under argon atmosphere. Upon completion, volatile components were removed under reduced pressure. To the crude mixture was added $\mathrm{CH}_{2} \mathrm{Cl}_{2}(2.6 \mathrm{~mL})$, acetic anhydride $(74 \mu \mathrm{L}, 0.78$ $\mathrm{mmol}), \mathrm{Et}_{3} \mathrm{~N}(0.12 \mathrm{~mL}, 0.86 \mathrm{mmol})$, and DMAP $(8.6 \mathrm{mg}, 0.070 \mathrm{mmol})$. After $3 \mathrm{~h}$, volatiles were removed and the product was purified by silica gel chromatography (99:1 pentane/ethyl ether plus $1 \%$ triethylamine) to give the seven-membered glycal $19(71.1 \mathrm{mg}, 45 \%)$ as a clear oil.

Method B (with DABCO as base and toluene as solvent): A flame-dried Schlenk flask, reflux condenser, and stir bar was charged with alkynyl diol $18(95.2 \mathrm{mg}, 0.476 \mathrm{mmol}$, azeotropically dried from toluene), tungsten hexacarbonyl $(25.1 \mathrm{mg}, 0.0713 \mathrm{mmol})$ and, freshly sublimed DABCO (107.7 mg, $0.9601 \mathrm{mmol})$. The mixture was dissolved in toluene $(2.4 \mathrm{~mL})$ and irradiated at $350 \mathrm{~nm}$ for $6 \mathrm{~h}$ at $70{ }^{\circ} \mathrm{C}$ under argon atmosphere. Upon completion, volatile components were removed under reduced pressure. To the crude mixture was added $\mathrm{CH}_{2} \mathrm{Cl}_{2}(1.9$ $\mathrm{mL})$, followed by acetic anhydride $(67.9 \mu \mathrm{L}, 0.0720 \mathrm{mmol}), \mathrm{Et}_{3} \mathrm{~N}(0.1143 \mathrm{~mL}, 0.8201 \mathrm{mmol})$, and catalytic DMAP $(5.9 \mathrm{mg}, 0.048 \mathrm{mmol})$. After $3 \mathrm{~h}$, volatiles were removed and the product was purified by silica gel chromatography (99:1 pentane/ethyl ether plus $1 \%$ triethylamine) to give the seven-membered glycal $19(79.7 \mathrm{mg}, 69 \%)$ as a clear oil.

$[\alpha]^{23}{ }_{\mathrm{D}}=-11.1\left(\mathrm{CHCl}_{3}, \mathrm{c}=2.14\right)$; IR(neat): 2988, 2938, 1745, 1643, 1453, 1373, 1231, 1177, 1088, 1038, 867, $740 \mathrm{~cm}^{-1} ;{ }^{1} \mathrm{H}$ NMR $\left(600 \mathrm{MHz}, \mathrm{CDCl}_{3}\right) \delta 6.31(\mathrm{dd}, J=5.9,2.1 \mathrm{~Hz}, 1 \mathrm{H}) ; 5.40$ (dd, $J=5.7,2.4 \mathrm{~Hz}, 1 \mathrm{H}) ; 4.95(\mathrm{dd}, J=9.1,7.1 \mathrm{~Hz}, 1 \mathrm{H}) ; 4.51$ (dt, $J=9.1,2.4,1.9 \mathrm{~Hz}, 1 \mathrm{H}) ; 3.73$ (pentet, $J=6.7 \mathrm{~Hz}, 1 \mathrm{H}) ; 3.69(\mathrm{t}, J=9.1 \mathrm{~Hz}, 1 \mathrm{H}) ; 2.10(\mathrm{~s}, 3 \mathrm{H}) ; 1.40(\mathrm{~s}, 3 \mathrm{H}) ; 1.39$ (s, 3H); 1.27 (d, 
$J=6.7 \mathrm{~Hz}, 3 \mathrm{H}) ;{ }^{13} \mathrm{C} \mathrm{NMR}\left(150 \mathrm{MHz}, \mathrm{CDCl}_{3}\right) \delta 170.1,146.2,114.1,109.9,79.8,77.9,77.0$, 73.9, 27.1, 26.9, 21.3, 18.3; Anal. Calcd for $\mathrm{C}_{12} \mathrm{H}_{18} \mathrm{O}_{5}: \mathrm{C}, 59.49$; H, 7.49. Found: C, 59.61; H, 7.67 .

$\operatorname{COSY}\left({ }^{1} \mathrm{H}-{ }^{1} \mathrm{H}\right)$ spectrum of $19\left(600 \mathrm{MHz}, \mathrm{CDCl}_{3}\right)$ :

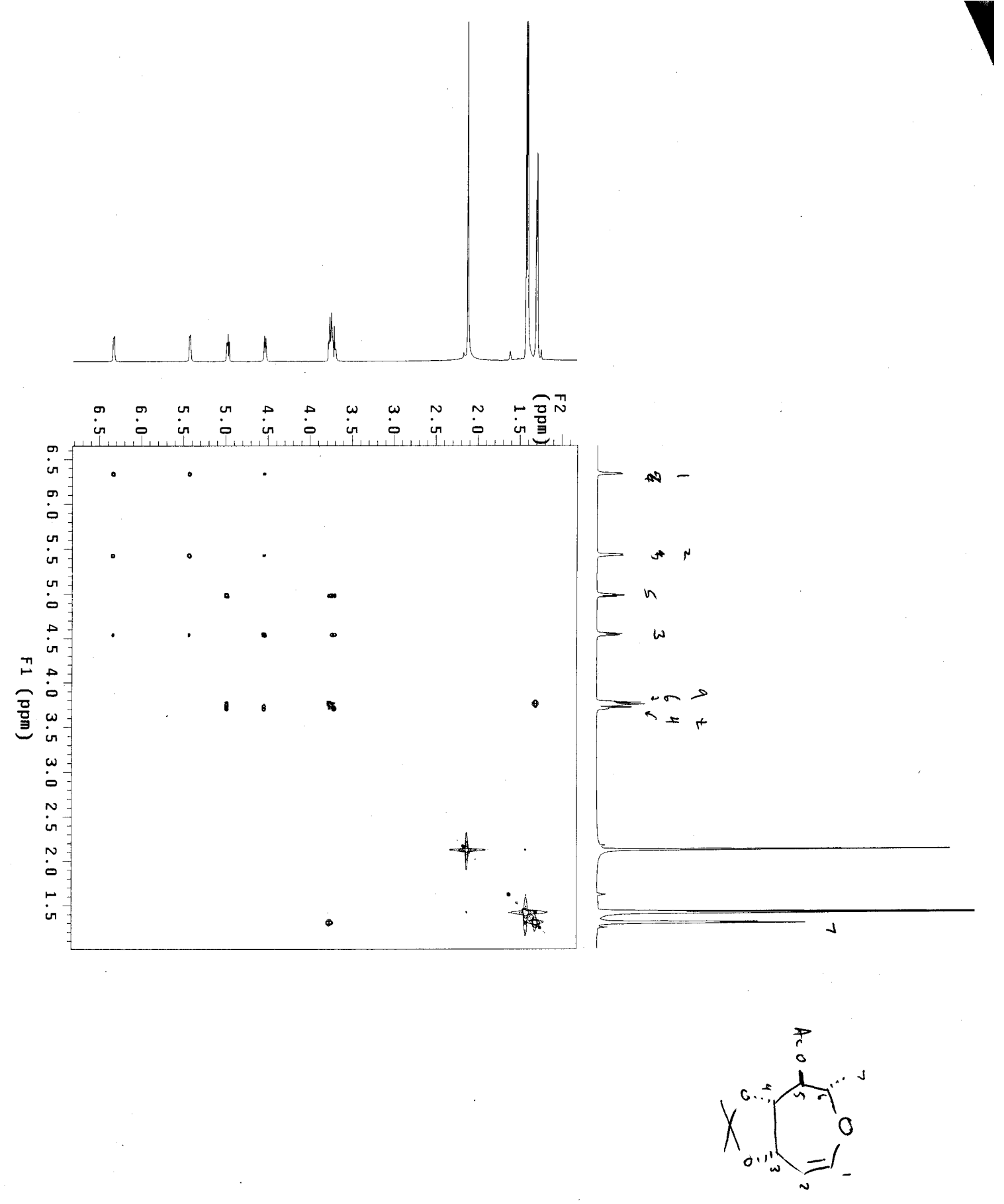




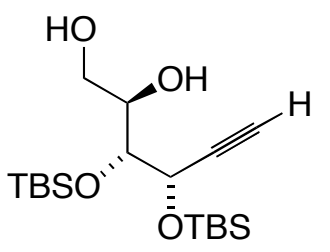

22

IR (neat): 3447, 3312, 2955, 2930, 2888, 2859, 1472, 1362, 1255, 1098, 885, $838 \mathrm{~cm}^{-1} ;{ }^{1} \mathrm{H}$ NMR $\left(400 \mathrm{MHz}, \mathrm{CDCl}_{3}\right) \delta 4.54(\mathrm{dd}, J=4.8,2.2 \mathrm{~Hz}, 1 \mathrm{H}) ; 4.09$ (pentet, $\left.J=3.7 \mathrm{~Hz}, 1 \mathrm{H}\right) ; 3.76-3.70$ (mult, 3H); 2.47 (d, $J=2.2 \mathrm{~Hz}, 1 \mathrm{H}) ; 0.92(\mathrm{~s}, 9 \mathrm{H}) ; 0.90(\mathrm{~s}, 9 \mathrm{H}) ; 0.20$ (s, 3H); 0.16 (s, 3H); 0.13 $(\mathrm{s}, 3 \mathrm{H}) ; 0.11(\mathrm{~s}, 3 \mathrm{H}) ;{ }^{13} \mathrm{C} \mathrm{NMR}\left(100 \mathrm{MHz}, \mathrm{CDCl}_{3}\right) \delta 81.6,75.2,73.0,71.9,67.7,63.4,25.8$, 18.2, 18.1, -4.6, -4.7, -5.2; Anal. Calcd for $\mathrm{C}_{18} \mathrm{H}_{38} \mathrm{O}_{4}$ : C, 57.70; H, 10.22. Found: C, 57.60; H, 10.33 .

5-tert-butyldimethylsiloxy-6-hydroxymethyl-oxa-2,4-cyclohexadiene (24)

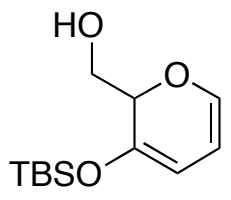

24

IR (neat): 3337, 2930, 2888, 2859, 1647, 1605, 1472, 1256, 1050, 984, 909, 839, $781 \mathrm{~cm}^{-1} ;{ }^{1} \mathrm{H}$ NMR $\left(300 \mathrm{MHz}, \mathrm{CDCl}_{3}\right) \delta 6.20(\mathrm{dd}, J=17.2,10.7 \mathrm{~Hz}, 1 \mathrm{H}) ; 5.42(\mathrm{~d}, J=17.5 \mathrm{~Hz}, 1 \mathrm{H}) ; 5.11(\mathrm{~d}, J$ $=10.8 \mathrm{~Hz}, 1 \mathrm{H}) ; 5.06(\mathrm{t}, J=7.2 \mathrm{~Hz}, 1 \mathrm{H}) ; 4.24(\mathrm{~d}, J=7.0 \mathrm{~Hz}, 2 \mathrm{H}) ; 1.01(\mathrm{~s}, 9 \mathrm{H}) ; 0.13(\mathrm{~s}, 6 \mathrm{H})$. 
$\operatorname{COSY}\left({ }^{1} \mathrm{H}-{ }^{1} \mathrm{H}\right)$ spectrum of $24\left(400 \mathrm{MHz}, \mathrm{CDCl}_{3}\right)$ :

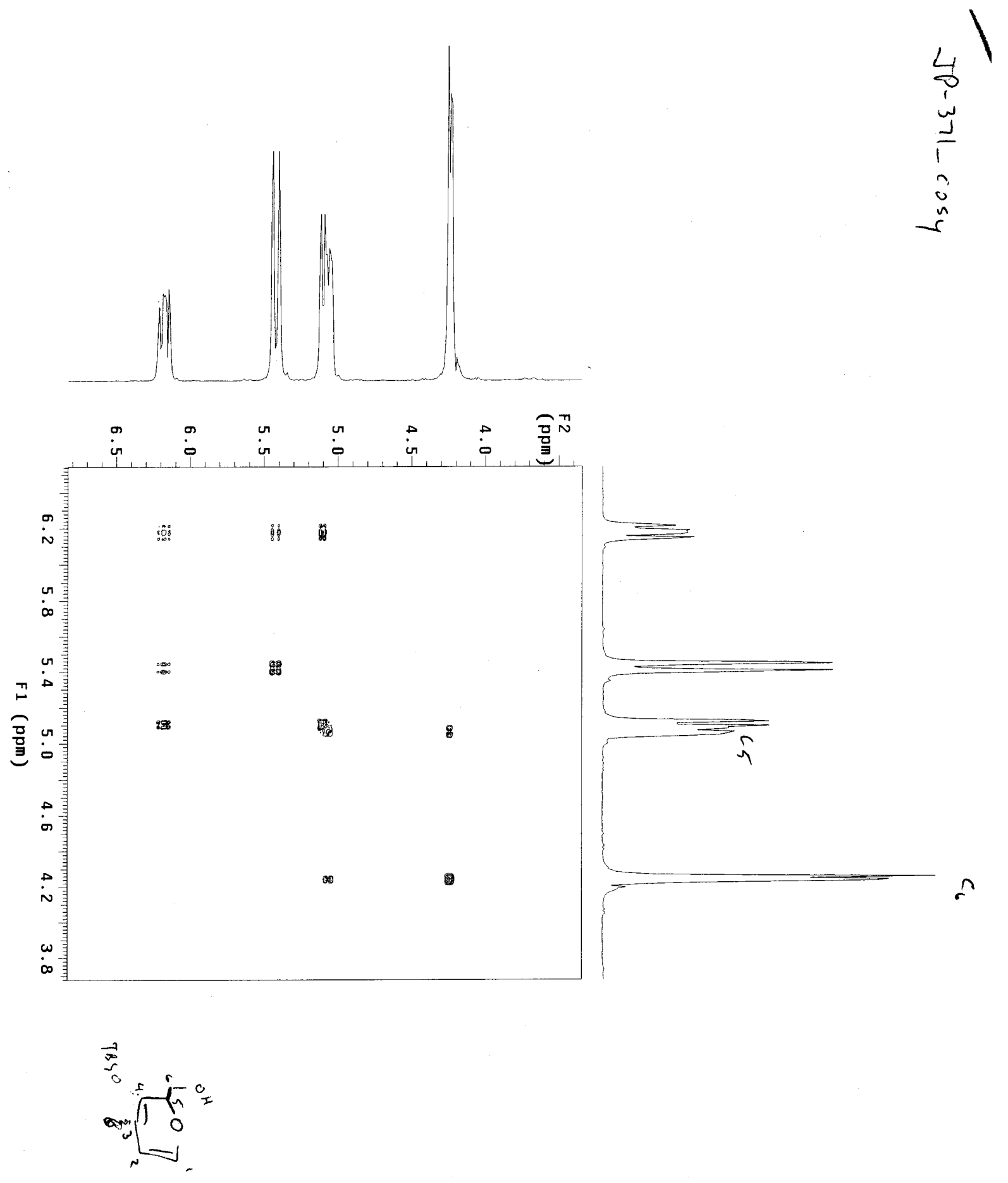

